\title{
ON ORTHOGONALLY ADDITIVE FUNCTIONS WITH BIG GRAPHS
}

\author{
KAROL BARON
}

\begin{abstract}
Let $E$ be a separable real inner product space of dimension at least 2 and $V$ be a metrizable and separable linear topological space. We show that the set of all orthogonally additive functions mapping $E$ into $V$ and having big graphs is dense in the space of all orthogonally additive functions from $E$ into $V$ with the Tychonoff topology.
\end{abstract}

Let $E$ be a real inner product space of dimension at least 2 and $V$ a linear topological Hausdorff space. A function $f$ mapping $E$ into $V$ is called orthogonally additive, if

$$
f(x+y)=f(x)+f(y) \quad \text { for all } x, y \in E \text { with } x \perp y .
$$

It is well known, see [6, Corollary 10] (cf. also [4, Theorem 1]), that every orthogonally additive function $f$ defined on $E$ has the form

$$
f(x)=a\left(\|x\|^{2}\right)+b(x) \quad \text { for } x \in E,
$$

where $a$ and $b$ are additive functions uniquely determined by $f$.

We continue a study of topological properties of some sets of orthogonally additive functions.

Given a non-empty set $S$ consider the set $V^{S}$ of all functions from $S$ into $V$ with the usual Tychonoff topology; clearly $V^{S}$ is a linear topological space.

Received: 29.07.2016. Accepted: 15.09.2016. Published online: 18.12.2016.

(2010) Mathematics Subject Classification: 39B55, 39B52, 54C10.

Key words and phrases: orthogonal additivity, inner product space, linear topological space, Tychonoff topology, big graph, dense set. 
In what follows we consider $\operatorname{Hom}_{\perp}(E, V)$ and $\operatorname{Hom}(S, V)$ for $S \in\{\mathbb{R}, E\}$ with the topology induced by $V^{E}$ and $V^{S}$, respectively, where

$$
\operatorname{Hom}_{\perp}(E, V)=\{f: E \rightarrow V \mid f \text { is orthogonally additive }\}
$$

and

$$
\operatorname{Hom}(S, V)=\{f: S \rightarrow V \mid f \text { is additive }\}
$$

for $S \in\{\mathbb{R}, E\}$. According to [2, Corollary 1]:

1. If $V \neq\{0\}$, then $\operatorname{Hom}(E, V)$ is closed and nowhere dense in $\operatorname{Hom}_{\perp}(E, V)$.

This and [1, Theorem] gives:

2. The set

$$
\left\{f \in \operatorname{Hom}_{\perp}(E, V): f \text { is injective and } f(E)=V\right\}
$$

is nowhere dense in $\operatorname{Hom}_{\perp}(E, V)$.

Moreover, see [3, Corollaries 2.3 and 2.4]:

3. If card $E \leq \operatorname{card} V$, then the set

$$
\left\{f \in \operatorname{Hom}_{\perp}(E, V): f \text { is injective and } f(E) \neq V\right\}
$$

is dense in $\operatorname{Hom}_{\perp}(E, V)$.

4. If $\operatorname{card} V \leq \operatorname{card} E$, then the set

$$
\left\{f \in \operatorname{Hom}_{\perp}(E, V): f(E)=V \text { and } f \text { is not injective }\right\}
$$

is dense in $\operatorname{Hom}_{\perp}(E, V)$.

The main result of this note concerns density of the set of all orthogonally additive functions with big graphs. Following [5, p. 317] we say that a function $f$ mapping a topological space $X$ into a topological space $Y$ has a big graph if $B \cap \operatorname{Graph}(f) \neq \emptyset$ for every Borel subset $B$ of $X \times Y$ such that the projection $\pi_{X}(B)$ of $B$ onto $X$ has the cardinality card $X$. We start with the following theorem.

THEOREM 1. If $\mathcal{R}$ is a family of subsets of $E \times V$ such that

$$
\operatorname{card} \pi_{E}(R)>\aleph_{0} \quad \text { and } \quad \operatorname{card} \pi_{E}(R) \geq \operatorname{card} \mathcal{R} \quad \text { for } R \in \mathcal{R},
$$

then the set

$$
\left\{f \in \operatorname{Hom}_{\perp}(E, V): R \cap \operatorname{Graph}(f) \neq \emptyset \text { for every } R \in \mathcal{R}\right\}
$$

is dense in $\operatorname{Hom}_{\perp}(E, V)$. 
Proof. Since (see [2, Theorem 1]) the operator which assigns the function $f$ defined by (1) to the variable $(a, b)$ from $\operatorname{Hom}(\mathbb{R}, V) \times \operatorname{Hom}(E, V)$ is a homeomorphism onto $\operatorname{Hom}_{\perp}(E, V)$, it is enough to prove that the set

(2) $\bigcap_{R \in \mathcal{R}} \bigcup_{x \in E}\left\{(a, b) \in \operatorname{Hom}(\mathbb{R}, V) \times \operatorname{Hom}(E, V):\left(x, a\left(\|x\|^{2}\right)+b(x)\right) \in R\right\}$

is dense in $\operatorname{Hom}(\mathbb{R}, V) \times \operatorname{Hom}(E, V)$.

We shall show more: if $a \in \operatorname{Hom}(\mathbb{R}, V)$ and $\mathcal{U} \subset \operatorname{Hom}(E, V)$ is open and non-empty, then $\{a\} \times \mathcal{U}$ intersects set (2). To this end we may assume that

$$
\mathcal{U}=\left\{b \in \operatorname{Hom}(E, V): b\left(z_{n}\right) \in U_{n} \text { for } n \in\{1, \ldots, N\}\right\}
$$

with some open subsets $U_{1}, \ldots, U_{N}$ of $V, z_{1}, \ldots, z_{N} \in E$ and $N \in \mathbb{N}$.

Fix $b_{0} \in \mathcal{U}$. To prove that $\{a\} \times \mathcal{U}$ meets set (2) it is enough to show that there is a $b \in \operatorname{Hom}(E, V)$ such that $(a, b)$ is in the set (2) and

$$
b\left(z_{n}\right)=b_{0}\left(z_{n}\right) \text { for } n \in\{1, \ldots, N\} .
$$

Let $H$ be a base of the vector space $E$ over the field $\mathbb{Q}$ of all rationals and let $H_{0}$ be a finite subset of $H$ such that $z_{1}, \ldots, z_{N} \in \operatorname{Lin}_{\mathbb{Q}} H_{0}$. Put

$$
\gamma=\operatorname{card} \mathcal{R}
$$

and let $\left(R_{\alpha}\right)_{\alpha<\gamma}$ be a transfinite sequence of all elements of $\mathcal{R}$. (We treat $\gamma$ as an ordinal.) By transfinite induction we define an injective transfinite sequence $\left(x_{\alpha}\right)_{\alpha<\gamma}$ of vectors of $E$ and a transfinite sequence $\left(v_{\alpha}\right)_{\alpha<\gamma}$ of vectors of $V$ such that for every $\alpha<\gamma$ we have

$$
\begin{gathered}
H_{0} \cup\left\{x_{\beta}: \beta \leq \alpha\right\} \text { is linearly independent over } \mathbb{Q}, \\
H_{0} \cap\left\{x_{\beta}: \beta \leq \alpha\right\}=\emptyset,
\end{gathered}
$$

and

$$
\left(x_{\alpha}, v_{\alpha}\right) \in R_{\alpha} .
$$

We proceed as follows. If $\delta<\gamma,\left(x_{\alpha}\right)_{\alpha<\delta}$ is an injective transfinite sequence of vectors of $E$ and $\left(v_{\alpha}\right)_{\alpha<\delta}$ is a transfinite sequence of vectors of $V$ such that (4) - (6) hold for every $\alpha<\delta$, then

$$
\begin{aligned}
\operatorname{card} \operatorname{Lin}_{\mathbb{Q}}\left(H_{0} \cup\left\{x_{\alpha}: \alpha<\delta\right\}\right) & \leq \aleph_{0} \cdot \max \left\{\aleph_{0}, \operatorname{card} \delta\right\} \\
& =\max \left\{\aleph_{0}, \operatorname{card} \delta\right\}<\operatorname{card} \pi_{E}\left(R_{\delta}\right),
\end{aligned}
$$


and so the set

$$
\pi_{E}\left(R_{\delta}\right) \backslash \operatorname{Lin}_{\mathbb{Q}}\left(H_{0} \cup\left\{x_{\alpha}: \alpha<\delta\right\}\right)
$$

is non-empty; choosing a point $x_{\delta}$ from this set we see that $H_{0} \cup\left\{x_{\alpha}: \alpha \leq \delta\right\}$ consists of vectors linearly independent over $\mathbb{Q}$ and $\left(x_{\delta}, v_{\delta}\right) \in R_{\delta}$ for some $v_{\delta} \in V$.

Let $b: E \rightarrow V$ be an additive function such that

$$
\left.b\right|_{H_{0}}=\left.b_{0}\right|_{H_{0}} \quad \text { and } \quad b\left(x_{\alpha}\right)=v_{\alpha}-a\left(\left\|x_{\alpha}\right\|^{2}\right) \quad \text { for } \alpha<\gamma .
$$

Then (3) holds and

$$
\left(x_{\alpha}, a\left(\left\|x_{\alpha}\right\|^{2}\right)+b\left(x_{\alpha}\right)\right)=\left(x_{\alpha}, v_{\alpha}\right) \in R_{\alpha}
$$

for $\alpha<\gamma$ and so $(a, b)$ is in the set (2).

COROllary 1. If $E$ is separable and $V$ is metrizable and separable, then the set

$$
\left\{f \in \operatorname{Hom}_{\perp}(E, V): f \text { has a big graph }\right\}
$$

is dense in $\operatorname{Hom}_{\perp}(E, V)$.

Proof. If $\mathcal{R}$ denotes the family

$$
\left\{B \subset E \times V: B \text { is Borel and card } \pi_{E}(B)=\operatorname{card} E\right\}
$$

then (see, e.g., [5, Theorem 2.3.4])

$$
\operatorname{card} \mathcal{R}=\mathfrak{c}=\operatorname{card} E=\operatorname{card} \pi_{E}(B)
$$

for every $B \in \mathcal{R}$.

Functions with big graphs have a lot of interesting properties. In particular the following (see [5, Theorems 2.5.6 and 2.8.3] and the proofs of Theorems 12.4.4 and 12.4.5 in [5]):

5. Assume $X$ and $Y$ are Polish spaces.

(i) If $X$ has no isolated points, then the graph of any function with big graph mapping $X$ into $Y$ is dense in $X \times Y$.

(ii) If $X$ and $Y$ have no isolated points and $f: X \rightarrow Y$ has a big graph, then neither $\operatorname{Graph}(f)$, nor $(X \times Y) \backslash \operatorname{Graph}(f)$ contains a second category set with the Baire property. 
(iii) If $X$ and $Y$ are connected, then the graph of any function with big graph mapping $X$ into $Y$ is connected.

Hence and from Corollary 1 we obtain the following corollary.

Corollary 2. If $E$ is separable and Hilbert and $V$ is Polish, $V \neq\{0\}$, then the set

$$
\begin{aligned}
\left\{f \in \operatorname{Hom}_{\perp}(E, V):\right. & \operatorname{Graph}(f) \text { is dense and connected, } \\
& \text { and neither } \operatorname{Graph}(f), \text { nor }(E \times V) \backslash \operatorname{Graph}(f) \\
& \text { contains a second category set with the Baire property\} }
\end{aligned}
$$

is dense in $\operatorname{Hom}_{\perp}(E, V)$.

At the end note also that:

6. If $V \neq\{0\}$, then the complement of the set (7) is dense in $\operatorname{Hom}_{\perp}(E, V)$.

In fact, an obvious modification of the proof of Theorem 1 shows that:

7. The set

$$
\left\{f \in \operatorname{Hom}_{\perp}(E, V): f(E) \text { is countable }\right\}
$$

is dense in $\operatorname{Hom}_{\perp}(E, V)$.

The reader interested in further problems connected with orthogonal additivity is referred to the survey paper [7] by Justyna Sikorska.

Acknowledgement. The research was supported by the University of Silesia Mathematics Department (Iterative Functional Equations and Real Analysis program).

\section{References}

[1] Baron K., Orthogonally additive bijections are additive, Aequationes Math. 89 (2015), 297-299.

[2] Baron K., On the continuous dependence of solutions to orthogonal additivity problem on given functions, Ann. Math. Sil. 29 (2015), 19-23.

[3] Baron K., On orthogonally additive injections and surjections, Comment. Math. 55 (2015), 157-162.

[4] Baron K., Rätz J., On orthogonally additive mappings on inner product spaces, Bull. Polish Acad. Sci. Math. 43 (1995), 187-189.

[5] Kuczma M., An introduction to the theory of functional equations and inequalities. Cauchy's equation and Jensen's inequality, Second edition (edited by A. Gilanyi), Birkhäuser Verlag, Basel, 2009. 
[6] Rätz J., On orthogonally additive mappings, Aequationes Math. 28 (1985), 35-49.

[7] Sikorska J., Orthogonalities and functional equations, Aequationes Math. 89 (2015), $215-277$.

Institute of Mathematics

University of Silesia

BANKOWA 14

40-007 Katowice

POLAND

e-mail: baron@us.edu.pl 